\title{
Modeling homing suppression gene drive in haplodiploid organisms
}

\author{
Yiran Liu ${ }^{1}$, Jackson Champer ${ }^{1 *}$ \\ ${ }^{1}$ Center for Bioinformatics, School of Life Sciences, Peking-Tsinghua Center for Life Sciences, \\ Peking University, Beijing, China 100871 \\ *jchamper@pku.edu.cn
}

\begin{abstract}
Gene drives have shown great promise for suppression of pest populations. These engineered alleles can function by a variety of mechanisms, but the most common is the CRISPR homing drive, which converts wild-type alleles to drive alleles in the germline of heterozygotes. Some potential target species are haplodiploid, in which males develop from unfertilized eggs and thus have only one copy of each chromosome. This prevents drive conversion, a substantial disadvantage compared to diploids where drive conversion can take place in both sexes. Here, we study the characteristics of homing suppression gene drives in haplodiploids and find that a drive targeting a female fertility gene could still be successful. However, such drives are less powerful than in diploids. They are substantially more vulnerable to high resistance allele formation in the embryo due to maternally deposited Cas9 and gRNA and also to somatic cleavage activity. Examining models of continuous space where organisms move over a landscape, we find that haplodiploid suppression drives surprisingly perform nearly as well as in diploids, possibly due to their ability to spread further before inducing strong suppression. Together, these results indicate that gene drive can potentially be used to effectively suppress haplodiploid populations.
\end{abstract}




\section{Introduction}

Suppression gene drives have recently shown great promise for a variety of applications, in particular elimination of pest populations ${ }^{1-7}$. These genetic elements bias their own inheritance to increase in frequency in a population, eventually causing suppression by biasing the sex ratio or rendering enough individuals sterile or nonviable. Though the most rapid progress has been in Anopheles mosquitoes for reduction of vector-borne disease ${ }^{8,9}$ (for which modification drives have also been developed ${ }^{10-12}$ ), suppression gene drives could also be used to remove invasive species $^{13}$ or agricultural pests ${ }^{14}$. Thus far, gene drives have been demonstrated in a variety of organisms, including yeast ${ }^{15-18}$, flies ${ }^{19-36}$, mice ${ }^{37}$, and plants ${ }^{37}$. Most of these have been homing types drives, where a nuclease, usually CRISPR/Cas9, cuts a wild-type chromosome at a site directed by its gRNA. The chromosome then undergoes homology-directed repair, which results in the drive alleles being copied to the target site. Since this occurs in the germline, the drive alleles will be inherited at an increased rate. However, if end-joining repair occurs instead, than the wild-type site can mutate into a resistance allele, which cannot be converted to a drive allele $^{32,38,39}$. These resistance alleles have the potential to slow or even stop the spread of a gene drive. The dynamics of such drives have been modeled extensively ${ }^{40-50}$, which is particularly important for predicting the outcome of a real-world drive release.

Though many possible applications of gene drives have been considered ${ }^{1-7}$, haplodiploid species have received little attention as possible targets. In these species, males develop from unfertilized eggs and thus only have one of each chromosome, which limits drive conversion in homing drives to females. So far, there has only been a single modeling studying assessing the possibilities for modification drives in haplodiploids ${ }^{51}$. The study concluded that homing drives could be effective, but they were always slower than in diploids, especially when drive performance was reduced. Though these results are promising in some situations, rapid suppression of haplodiploids is often desirable. This is especially true in well-known pest species such as invasive fire ants, which can cause human harm in addition to catastrophic damage to ecosystems and agricultural production ${ }^{52-54}$. In this species, CRISPR genome engineering has already been demonstrated ${ }^{55}$, bringing the possibility of developing a gene drive closer to reality. Other major haplodiploid pests that could potentially be the target of a suppression drive include certain bark beetles and thrips, which damage trees and potatoes, respectively.

However, several drive mechanisms in diploids would not function in haplodiploids, particularly those that bias sex ratio or cause infertility or nonviability in males or in both males and females. Two studies attempted to develop models for haplodiploid suppression drives based on homing drives, but these lacked power to cause complete suppression on their own ${ }^{56,57}$. Our initial assessments indicated that of the established drive systems, only homing drives targeting haplosufficient but essential female fertility genes could cause strong suppression. In this study, we investigate this drive in detail, including its properties such as rate of spread and especially its overall suppressive power, comparing it to more well-studied but similar suppression drives in diploids. We find that embryo resistance and somatic cleavage activity can substantially reduce 
the efficiency of haplodiploid suppression drives, but over a wide parameter range, its performance is still nearly as good as diploid drives.

Additionally, many recently modeling studies of gene drive suppression have revealed unexpected complexity in spatially explicit scenarios. These scenarios could take the form of abstract patches ${ }^{58}$, complex networks of linked panmictic populations ${ }^{59,60}$, or arenas with continuous space ${ }^{61,62}$. In these simulations, the gene drive is often initially successful, eliminating the population in a particular region. However, this region can then be recolonized by wild-type individuals. The gene drive is still present in adjacent areas and moves in shortly afterward, completing cycles of suppression and recolonization as the drive "chases" wild-type alleles. Because even slightly weaker drives are more prone to suffering from such outcomes ${ }^{61}$, a haplodiploid homing suppression drive might lose its ability to effectively suppress populations. We used our continuous space model to assess the performance of haplodiploid drives, but we found that it was only, on average, slightly more vulnerable to chasing than diploid drives of the same design and performance, still usually suppressing the population in a timely manner. In some areas of parameter space, the haplodiploid drive even had small advantages over the diploid version. These results indicate that homing suppression gene drives targeting female fertility genes are promising candidates for genetic control of haplodiploid pest species.

\section{Methods}

Suppression drive strategy. In haplodiploids, Females are derived from the diploid progeny, and unfertilized eggs become males. Due to this special inheritance in haplodiploid species, many previously developed suppression gene drives could not function properly because males have only a single chromosome. However, we found that homing drives targeting female fertility genes could be used for population suppression in this study, despite drive conversion functioning only in females.

In drive heterozygotes, the wild-type alleles will be converted into drive alleles in the germline through target cleavage followed by homology-directed repair using the drive as a template. With such a drive, we target a haplosufficient but essential female fertility gene, leading to sterility in female drive homozygotes. The increasing frequency of drive alleles will result in accumulation of sterile drive homozygous females among the population, which can ultimately cause the population collapse.

However, there are two possible repair mechanisms after DNA cleavage, homology-directed repair and end-joining. If the cleaved target site is repaired by the latter way, it may cause the site to be mutated, and such sites couldn't be cleaved by Cas 9 in the future due to sequence mismatch with the gRNA. Because these can no longer be converted to drive alleles, they are called "resistance alleles". Most resistance alleles result in a non-functional target gene, which we called "r2" alleles. Such alleles could contribute to female sterility but would also slow the drive. 
Functional "r1" resistance alleles, a more serious type, will almost always result in failure of a suppression drives. However, multiple gRNAs ${ }^{23,29}$ and conserved target $\operatorname{sites}^{8}$ could be used to avoid the formation of functional resistance alleles, so we did not model them in our simulations.

Simulation model. The forward-in-time genetic simulation framework SLiM (version 3.6) was used to perform all simulations ${ }^{63}$. In this study, we used code for diploids targeting X-linked loci to model haplodiploids since inheritance patterns in both situations are identical. The model is described in detail in a previous study ${ }^{61}$. In short, the sexually reproducing population in our model is 50,000 with discrete generations. We release gene drive heterozygous females and drive carrying males at a frequency of $1 \%$ of the total population after allowing the population to equilibrate for 10 generations.

For reproducing individuals in our model, the process of gene drive takes place independently in each gamete. The rate for wild-type alleles being converted into drive alleles in the germline of heterozygotes was determined by the drive conversion rate or "efficiency". The probability of a wild-type allele instead being converted into a resistance allele in drive heterozygous females was equal to the germline resistance allele formation rate, which we set as $0.5 *(1$-drive conversion rate) in our model so that half of remaining wild-type alleles become resistance alleles after drive conversion takes place. Embryo resistance was also taken into consideration. If the offspring's mother possesses a gene drive allele while the offspring has any remaining wildtype target sites, they might be converted into resistance allele due to persistence of maternally deposited Cas 9 and gRNA in the embryo. Our default parameters represented an efficient drive $e^{8,11,12,64}$, with $95 \%$ drive conversion rate.

Drives could also have reduced fitness from two sources. Direct fitness affects were specified by the main fitness parameter, which previously referred to the fitness relative to wild-type in drive homozygotes, with multiplicative fitness per drive allele. For haplodiploids, this parameter is directly used for males with one drive allele. It affects female fecundity directly, and fitness also proportionally reduces the chance that a male will be selected as a mate. Our default fitness value was 0.95. Negative fitness affects can also stem from somatic expression in female drive/wildtype heterozygotes ${ }^{8,64,65}$. Our default for this parameter is 1 , but we also investigated effects of varying this parameter, where it has the effect of multiplying the fecundity of drive/wild-type heterozygous females.

Panmictic population model. To determine if gene drive is feasible for haplodiploid suppression, we first implemented the drive in a panmictic population. Fertile females could pick a male to mate with at random, and then the equation $\omega^{\prime}{ }_{i}=\omega_{i}{ }^{*} \beta /[(\beta-1) \mathrm{N} / \mathrm{K}+1]$ is used to calculate the female fecundity, where $\omega_{i}$ is the baseline fecundity due to genotype, $\mathrm{N}$ is total population size, $\mathrm{K}$ is the population carrying capacity, and $\beta$ is the low-density growth rate (the fitness advantage individuals experience when unaffected by competition due to lack of close neighbors with a default value of 6). We assumed the number of offspring conform to a binomial 
distribution and then draw 50 independent offspring with survival $\rho=\omega_{i}^{\prime} / 25$. This means that a wild-type female will produce two offspring on average if the population is near its capacity.

The genetic load is one way to assess the reproductive burden the drive places on the population. It refers to the fractional reduction in population reproductive capacity compared to a similar wild-type population, thus measuring the strength with which a gene drive can suppress the population. It is equal to 1 - (actual population during the next time step) / (expected next generation population if all individuals were wild-type). To allow for accurate measurements of the genetic load when it is high, population suppression was artificially prevented by allowing fertile female to have more offspring than normal during simulations used to measure genetic load. In such genetic load simulations, the drop size of the gene drive heterozygotes was $20 \%$ of the total population. The simulation was allowed to run for 50 generations for the drive to reach its equilibrium frequency, and then the last 100 generations of the simulation were considered for calculation of the average genetic load.

Spatial model. To conduct a more realistic assessment of drive performance, the panmictic model was expanded to a model involving continuous space. The 50,000 initial wild-type individuals were confined into a 1 x 1 arena where we dropped 500 drive-heterozygous into the center after allowing the simulation to equilibrate for 10 generations. In the spatial model, the females were limited to choosing mates within a radius equal to the migration value parameter (with a default of 0.04), and the females would not be able to reproduce if there wasn't a male to mate with around that circle. To model local competition, we scaled the fecundity of female to $\omega^{\prime}{ }_{i}=\omega_{i}{ }^{*} \beta /\left[(\beta-1) \rho_{i} / \rho+1\right]$, where $\rho_{i}$ represented the number of other individuals around a female within a circle of radius 0.01 , and $\rho$ was the carrying density (equal to K/total area). Due to an abundance of resources and the reduced competition, the female could have more reproduction if she was in a low-density area. Offspring were distributed randomly around the mother at a distance drawn from a normal distribution with zero mean and standard deviation equal to the migration rate. The result of this process will produce an average displacement equal to migration rate $* \sqrt{\pi / 2}$. If the offspring was placed outside of the arena, their position is redrawn until they fall within the boundaries.

Simulations are forced to stop 1,000 generations after the drive release if the population or the drive allele was not eliminated earlier. In suppression drive, we often see the "chasing" phenomenon between wild-type and drive individuals after releasing drives into the population ${ }^{29}$. The population around the center where the suppression drive is dropped could be eliminated rapidly, but then recolonized by escaping wild-type individuals. At the peak of this process, population is low, but Green's coefficient (see below) is increased as well ${ }^{29}$. The escaped wildtype individuals produce more offspring because of the lack of competition, and soon they occupy more space, decreasing Green's coefficient in response while increasing total population. The drive is still present, however, resulting in cycles of suppression and recolonization that have the appearances of the drive "chasing" wild-type individuals across the landscape. It is the lower 
generation of the first local maximum in Green's coefficient and the first local minimum in the number of wild-type individuals where we consider a chase to have begun.

To quantify the spatial clustering degree of the population for analysis of chasing, we calculated

Green's coefficient $(G)$ of each generation through the equation $G=\left(\frac{s^{2}}{n}-1\right) /(N-1)$, where $N$ was the total population, $n$ represented the mean number of the individuals and $s^{2}$ was the variance of the counts. For convenience of calculating, we divided the arena where we modeled into an $8 \times 8$ grid and counted the wild-type homozygous of each square. It is noted that we only count the wild-type homozygote individuals in the estimation since this will more closely correlate with chasing ${ }^{29}$.

Data generation. Simulations were performed on the High-Performance Computing Platform of the Center for Life Science at Peking University. Python were used to analyze the data and prepare the figures. The SLiM script, raw data, and chasing movies are available on GitHub (https://github.com/jchamper/ChamperLab/Haplodiploid-Suppression-Modeling).

\section{Results}

Characteristics of haplodiploid suppression drives. To assess the potential of a female fertility homing suppression drive in haplodiploids, we first compared drive characteristics between haplodiploids and diploids in panmictic populations. Our default parameters approximately corresponded to a successfully constructed drive in Anopheles with reduced fitness $\operatorname{costs}^{8}$, representing highly efficient but somewhat imperfect systems. The suppression drive targets a female fertility gene, causing females without wild-type alleles to be sterile (Figure 1A). In diploids, wild-type alleles could be converted into drive alleles during the germline stage in both males and females (Figure 1A), so that the drive frequency could increase rapidly (Figure 1B). Males have only one chromosome in haplodiploids, so drive conversion can only be completed in females (Figure 1A), resulting in a slower rate of increase (Figure 1B). Thus, the haplodiploid drive could still eliminate the population, but this occurred later than in diploids (Figure 1C). 
A

Female fertility homing suppression drive

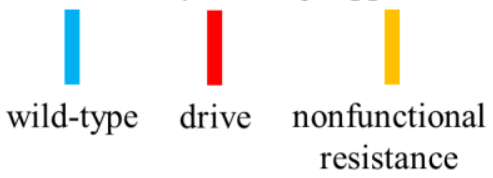

females with these genotypes are sterile
B

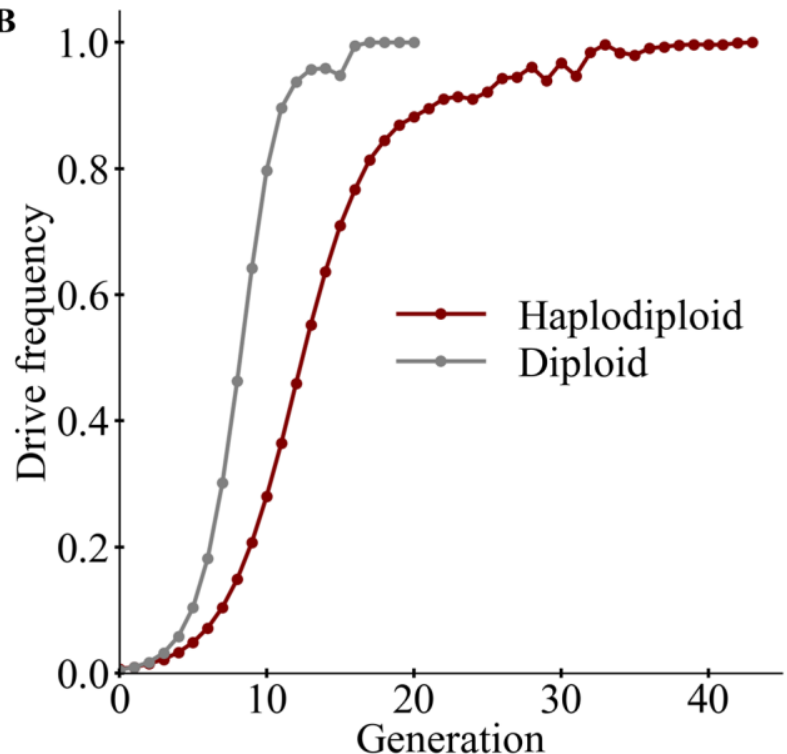

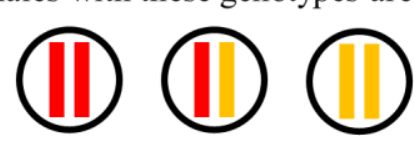

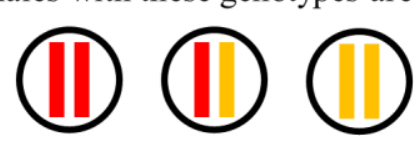

Drive conversion in male and female heterozygote germline cells

Diploid

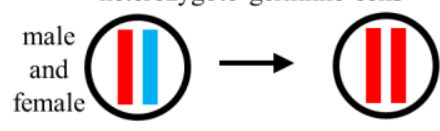

Drive conversion in female

heterozygote germline cells

C

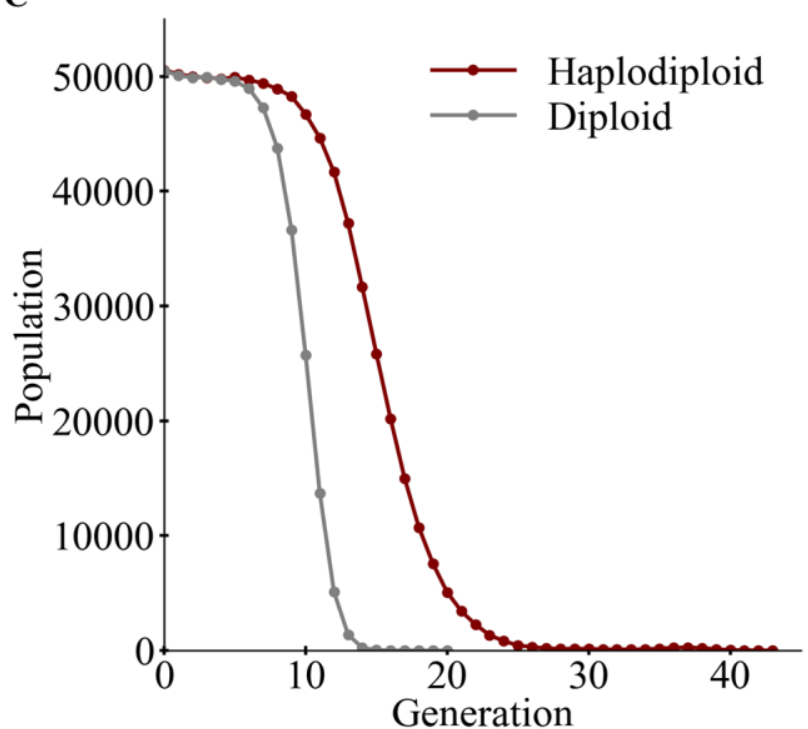

Figure 1 Homing suppression drives in haplodiploid and diploid populations. (A) In a female fertility homing drive, females with only drive alleles or nonfunctional resistance alleles are sterile. Such nonfunctional resistance alleles can be formed in the germline as an alternative to drive conversion or in the embryo from maternally deposited Cas9 and gRNA. The drive can also be impeded by somatic cleavage activity, which can reduce fertility in drive/wild-type heterozygotes. In diploids, the drive can increase in frequency quickly since drive conversion can take place in the germline of male and female heterozygotes. In haplodiploids, males form from unfertilized eggs and thus only have a single chromosome, allowing drive conversion only in females. (B,C) Drive heterozygotes (and drive males for the haplodiploid drive) with default performance characteristics were released at $1 \%$ frequency into a panmictic population of wild-type individuals. The $(\mathbf{B})$ drive allele frequency and $(\mathbf{C})$ population size were tracked for each generation. Displayed data is the average from 20 simulations.

To examine how drive performance parameters affect the rate at which the drives spread, we measured the haplodiploid drive allele frequency 16 generations after the drive individuals were released, which we found to give a wide spread of values, making it suitable for comparing drives with different performance. As expected, the drive conversion rate (the drive efficiency) and fitness were critical for rapid spread of the drive (Figure S1A). The germline resistance scarcely affected the suppression drive (Figure S1B), though it could only vary it from 0 to 0.05 due to high default drive efficiency. The drive allele frequency had a similar downward trend from increasing the embryo resistance allele formation rate (such alleles form due to maternally deposited Cas 9 and gRNA in the embryo) and the relative fitness from reduced female fertility 
due to somatic cleavage (Figure S1). This is because large reductions in the reproductive capacity of females (due to somatic cleavage eliminating wild-type alleles in some cells, which are required for fertility) or their offspring (due to embryo resistance alleles resulting in sterile daughters) can directly counterbalance drive conversion in females, preventing the drive from being able to increase in frequency.

Genetic load is a characteristic that measures suppressive power on a target population. It measures the reduction in reproductive capacity in the population compared to an equivalent wild-type population. A genetic load of 0 means that the drive does not affect reproduction, while a genetic load of 1 means that the drive has stopped all reproduction in the population. We measured the genetic load of the suppression drives after they had reached their long-term equilibrium frequency (Figure S2A). In general, the genetic load of diploid drives was higher than for haplodiploids (Figure 2), though the haplodiploid drive was still able to reach high genetic load at high efficiency (Figure S2B). Variation in drive efficiency (Figure 2A), fitness (Figure 2B), and germline resistance (Figure S2C) had similar effects on both drives. However, the genetic load in haplodiploids fell off drastically if the embryo resistance was more than 0.6 (Figure 2C) or if the relative fitness from somatic cleavage was less than 0.7 . This was not the case for diploid drives, though such drives would of course be slowed in these circumstances. 
A

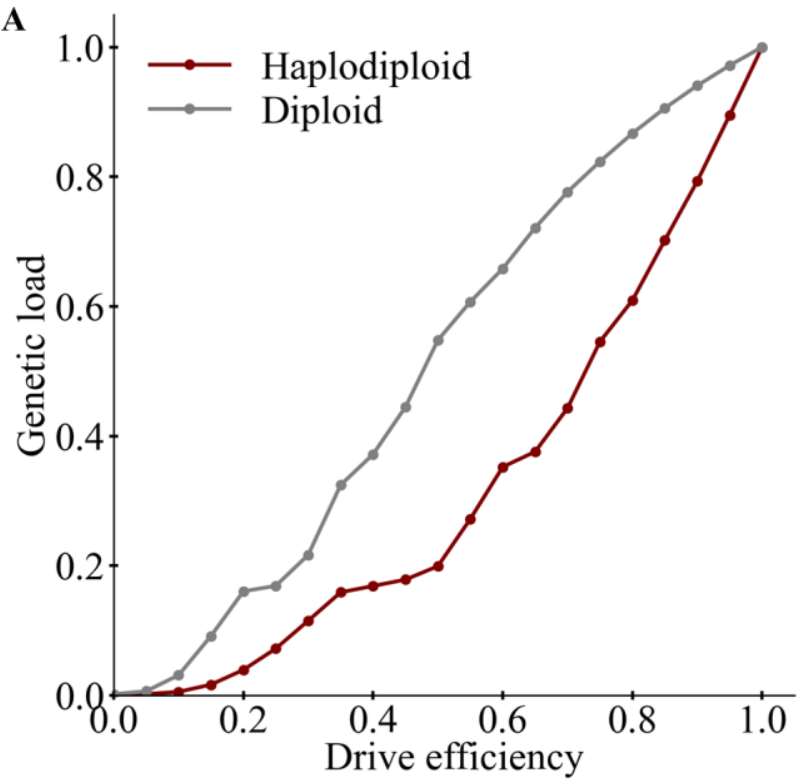

C

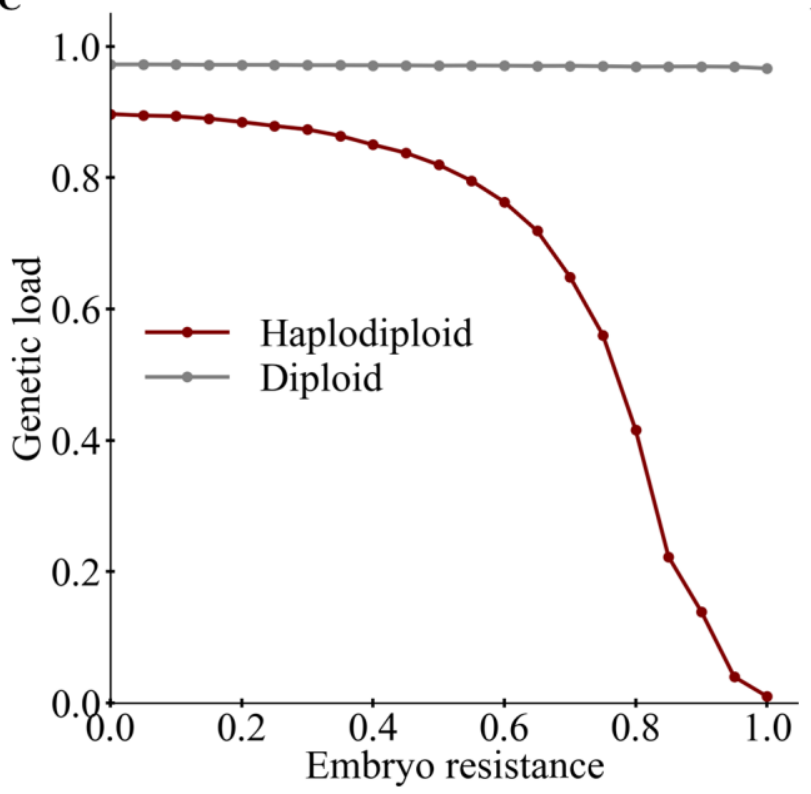

B

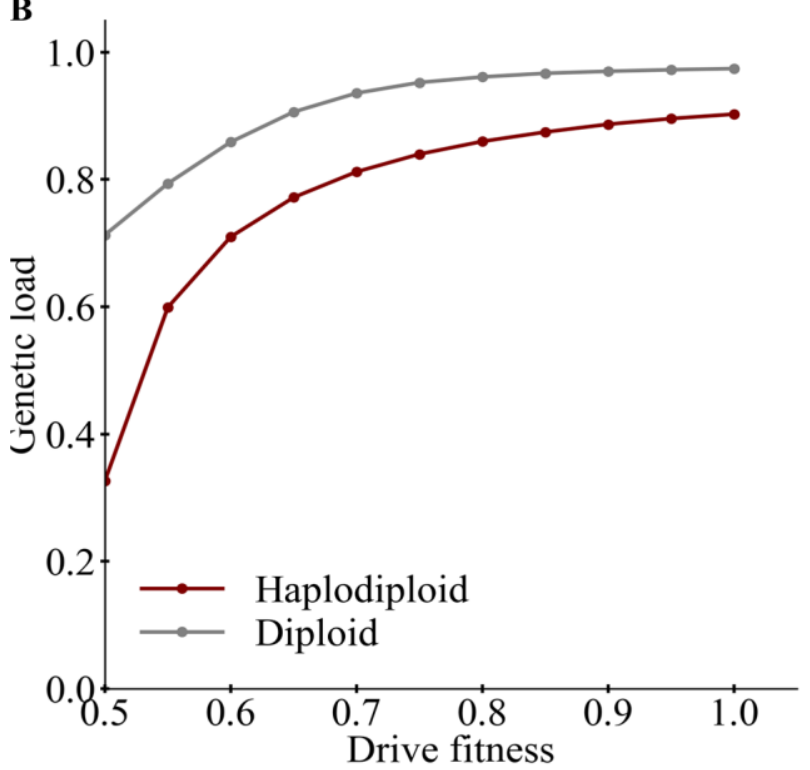

D

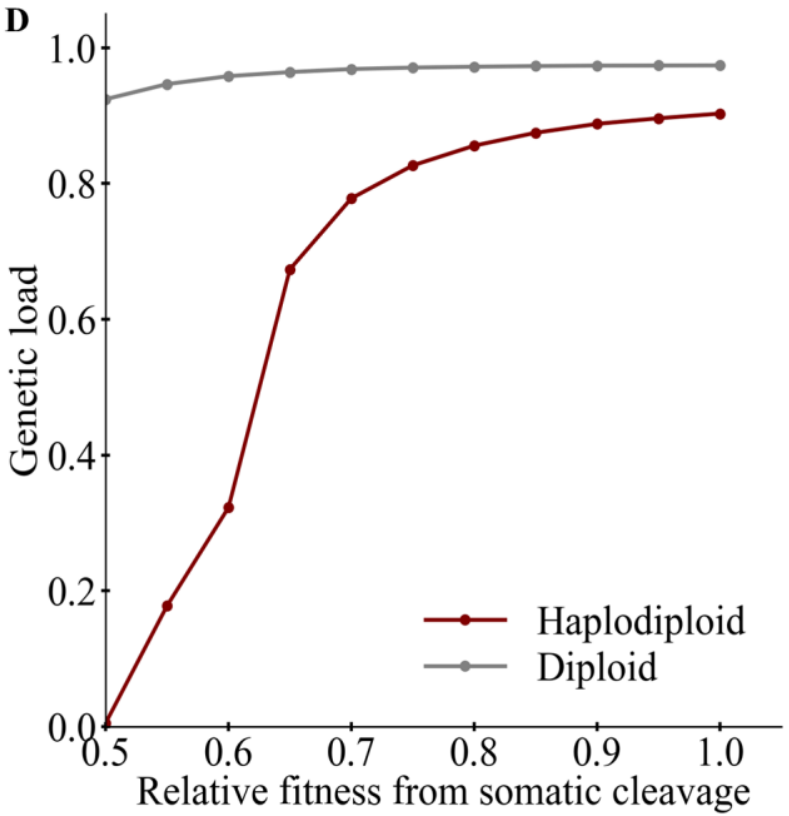

Figure 2 Effects of performance characteristics on the genetic load. The equilibrium genetic load is displayed for female fertility homing suppression drives with default performance characteristics and varying (A) drive conversion rate, (B) drive homozygote fitness, (C) embryo resistance allele formation rate, or (D) fitness cost in female heterozygotes due to somatic CRISPR cleavage activity. Displayed data is the average from 200 simulations.

Drive performance in continuous space. To examine drive performance in more realistic continuous space models, simulations were initialized by releasing drive heterozygous females and drive-carrying males into the center of a spatial population. These drive individuals initially represented $1 \%$ of the population, and the simulation was then allowed to develop for 1000 discrete generations. A new parameter for these simulations is the migration value, which controls the distance of mating distance and offspring placement. 
Previous studies indicated that suppression drives in continuous space could fail in several ways. In one, the genetic load would be insufficient to suppress the population, but because this also occurs in panmictic populations, we restricted our analysis to drives that would theoretically be able to suppress analogous panmictic populations. This included drives with at least $80 \%$ drive conversion rate and $80 \%$ fitness in homozygotes (with each drive allele contributing the square root of this fitness, and alleles having multiplicative fitness). A second mode of failure involves stochastic loss of the drive. This can also occur in panmictic populations, but it is far more common in models of suppression drives in continuous space ${ }^{61,66}$. However, we found that this outcome was very rate for a female fertility homing suppression gene drive in haplodiploids (Figure S3).

Another more common mode of drive failure was "chasing". This refers to a phenomenon in which some wild-type individuals escape from the drive and reach empty regions where the drive had previously eliminated the population ${ }^{61}$. Here, they experience reduced competition and are able to have high numbers of offspring. The drive remains in contact with these wild-type groups, "chasing" them and continuously causing suppression, but in many cases, the drive fails to ultimately eliminate the population, even after long periods of time. Chasing was quite common in our simulations of haplodiploids. Movies showing short and long chases involving the haplodiploid drive can be found on GitHub (https://github.com/jchamper/ChamperLab/Haplodiploid-Suppression-Modeling).

Specifically, when drive fitness, and more importantly, drive efficiency (representing the drive conversion rate), were high, chasing could sometimes be avoided, with the population rapidly suppressed (Figure 3A, Figure S4A-B). However, it was more common for a period of chasing to occur prior to suppression (Figure 3B). When both efficiency and fitness were low, chasing could persist indefinitely, with the population still in a chasing state after 1000 generations (Figure 3C). For simulations where chasing was followed by suppression, the period of chasing was usually fairly short (Figure 3D), but when efficiency was low, chasing could sometimes average several hundred generations.

In many cases, an outcome in which chasing persists could still provide benefits by reducing the population and its reproductive capacity. We measured this by tracking the average number of fertile females during a chase (Figure 3E). This indicated that even an inefficient drive could reduce the population several-fold compared to its initial state. However, long periods of chasing would increase the chance that functional resistance alleles could eventually develop, even if the rate of their formation would generally have been reduced to an acceptable level for panmictic populations in which suppression occurs quickly without chasing. This was previously demonstrated in diploid drives and would be similar for haplodiploid systems ${ }^{61}$. 

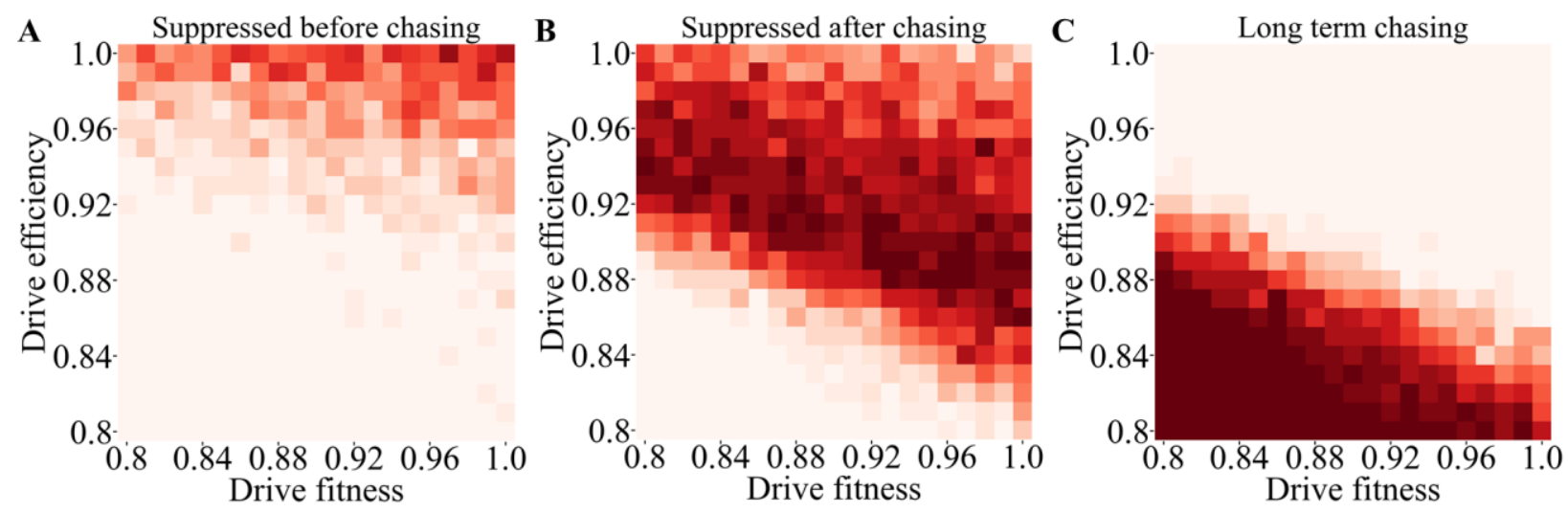

$\begin{array}{ccccc}0.00 & 0.25 & 0.50 & 0.75 & 1.00 \\ & \text { Frequency of outcome }\end{array}$

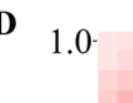

Duration of chasing

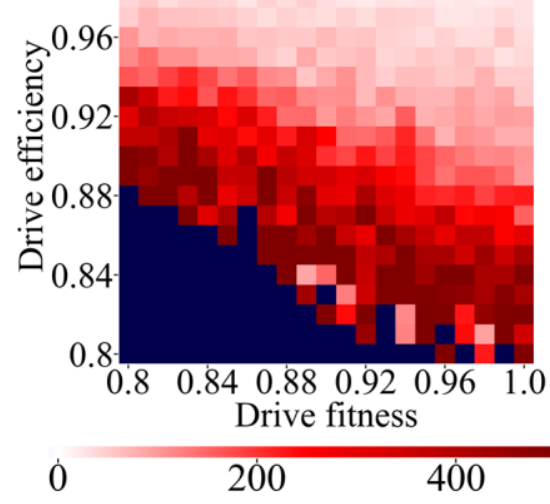

E Average number of fertile females

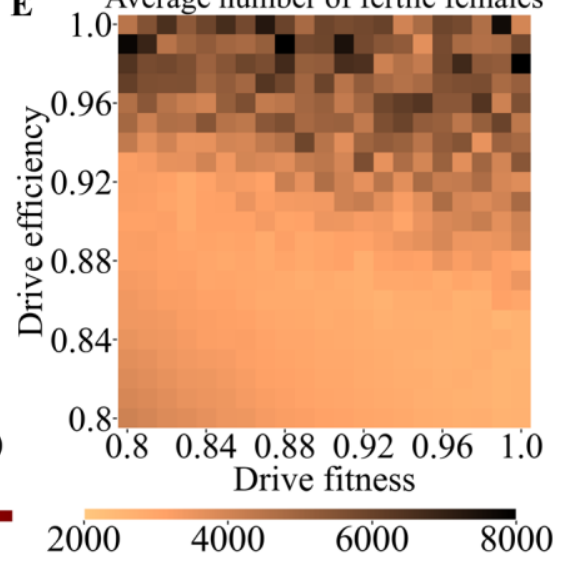

Figure 3 Outcomes of a drive release in continuous space with varying drive efficiency and fitness. Female drive heterozygotes and drive males with default parameters and varying efficiency and fitness were released in a 0.01 radius circle into the middle of a spatial population of 50,000 individuals and were tracked for 1,000 generations. Outcome rates are displayed for (A) suppression without chasing, (B) suppression after a period of chasing, and (C) simulations in which chasing was still occurring at the end of the simulation. Also displayed is (D) the average duration of chasing that eventually ended in suppression (blue represents parameter space where chasing did not end in any simulation), and (E) the average number of fertile females during any type of chasing. 20 simulations were assessed for each point in the parameter space.

Ecological characteristics can also influence the outcome of a suppression drive release. We thus varied the migration rate and the population growth rate at low densities, similarly tracking drive outcomes for an efficient drive (Figure S4C-D). Unlike most previously investigated drives, we found that the low-density growth rate had little effect in the range tested (Figure 4, Figure S4D) (though eventually, high enough low-density growth rates would yield equilibrium outcomes in all situations, including panmictic populations). Migration rate had a similar effect as drive efficiency. When migration was high, suppression before chasing was common (Figure 4A), followed by suppression after chasing for most of the parameter range (Figure 4B). When very low, long-term chasing outcomes were common (Figure 4C). However, most chasing was short and quickly ended in suppression at middle and high migration rates (Figure 4D). When migration was low, the average population during a chase was higher (Figure 4E). 

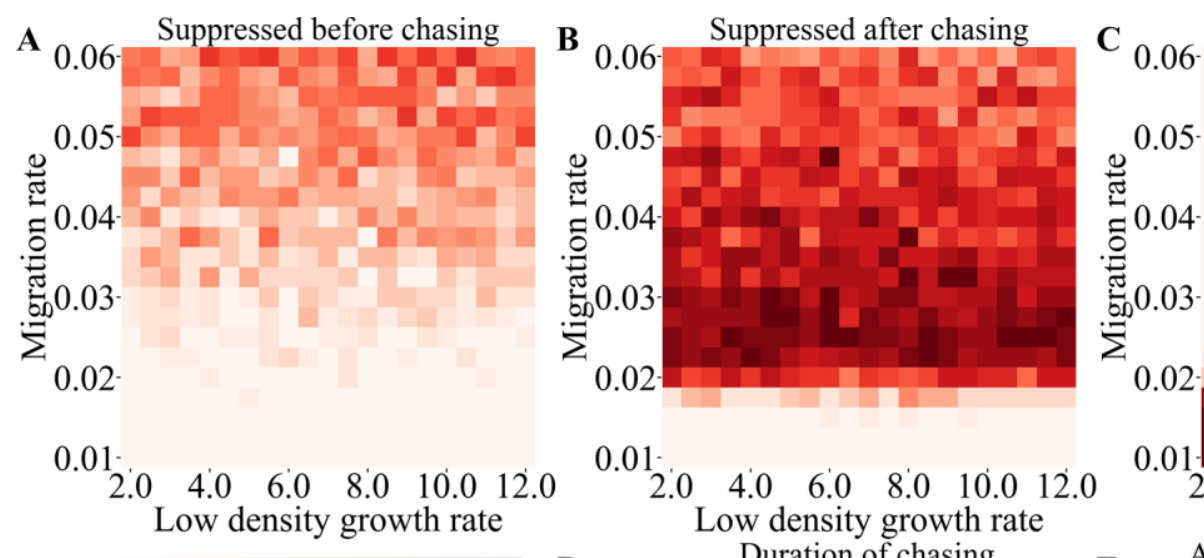
0.01
$\begin{array}{llllll}2.0 & 4.0 & 6.0 & 8.0 & 10.0 & 12.0\end{array}$ Low density growth rate $\begin{array}{cccc}0.00 & 0.25 & 0.50 & 0.75 \\ \text { Frequency of outcome }\end{array}$ Duration of chasing
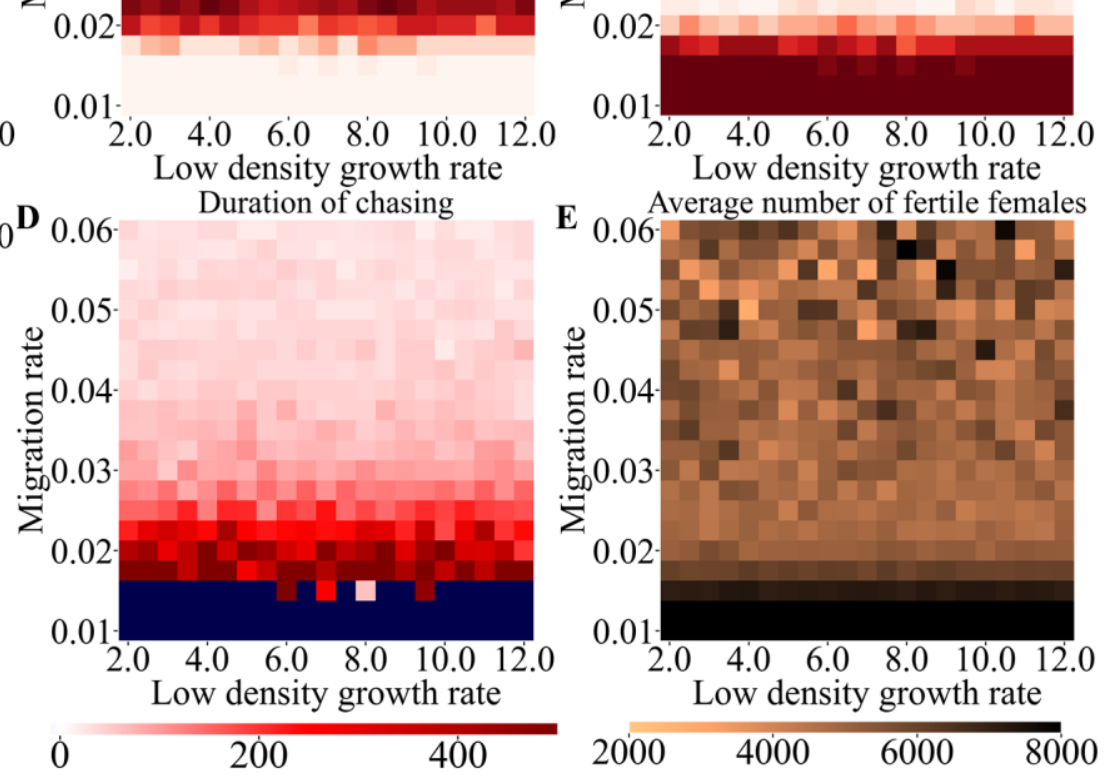

Long term chasing

$2000 \quad 4000 \quad 6000 \quad 8000$

Figure 4 Outcomes of a drive release in continuous space with varying migration rate and low-density growth rate. Female drive heterozygotes and drive males with default performance parameters and varying migration and low-density growth rate were released in a 0.01 radius circle into the middle of a spatial population of 50,000 individuals and were tracked for 1,000 generations. Outcome rates are displayed for (A) suppression without chasing, (B) suppression after a period of chasing, and (C) simulations in which chasing was still occurring at the end of the simulation. Also displayed is (D) the average duration of chasing that eventually ended in suppression (blue represents parameter space where chasing did not end in any simulation), and (E) the average number of fertile females during any type of chasing. 20 simulations were assessed for each point in the parameter space.

We also examined the effect of population density on haplodiploid drives, which was previously shown to affect certain types of drive systems ${ }^{61}$. However, like homing suppression systems in diploids, we found that population density had little effect on outcomes, except for slightly increased frequency of chasing at very low population density (Figure S5).

Comparison between haplodiploid and diploid drives. Our previous study showed that in diploids, a female fertility homing suppression drive was also vulnerable to the chasing phenomenon in continuous space, even though it was considered to be a good quality drive and performed better that both-sex sterile homing drives and driving Y chromosomes (based on Xshredders $)^{61}$. To measure how such a diploid drive compared to our haplodiploid drive, we compared their performance in identical conditions (Figure S6-7) with special attention to how performance varies with drive conversion efficiency (Figure 5). Despite the substantially higher genetic load and rate of increase of the diploid drive, both drives had very similar performance. 
Over a wide range of parameter space, the haplodiploid drive tended to have outcomes where chasing was followed by suppression (Figure S6B, S7B) when suppression would occur without chasing in the diploid drive (Figure S6A, S7A). However, in most of this range, the chases were very short (Figure 3D, 4D, 5), thus causing only small delays before complete suppression. In other areas of parameter space, the haplodiploid drive actually had some small advantages in avoiding chasing, especially when the drive was less efficient (Figure 5, S6C) or when the lowdensity growth rate was high (Figure S7C). Indeed, the haplodiploid drive in general was not substantially negatively affected by higher low-density growth rates, in contrast to other drives ${ }^{61}$ (though if this parameter becomes high enough, than any drive with a genetic load of less than 1 would eventually lose its ability to suppress the population and instead reach an equilibrium state). Also of note, the haplodiploid drive experienced drive loss much less than the diploid drive (Figure 5, S6D-E, S7D-E).

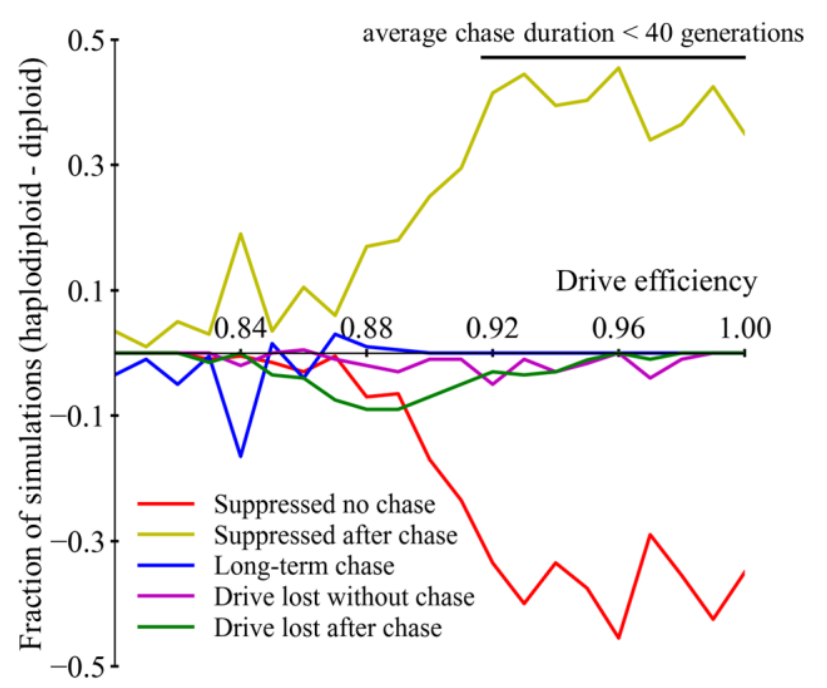

Figure 5 Comparison of outcomes in continuous space between haplodiploid and diploid suppression drives. Drive heterozygotes (and drive males for the haplodiploid drive) with default parameters and varying drive efficiency were released in a 0.01 radius circle into the middle of a spatial population of 50,000 individuals and were tracked for 1,000 generations. Differences in outcome rates between haplodiploids and diploids are displayed (higher values represent outcomes more common in haplodiploids). 200 simulations were assessed for each point in the parameter space. Data for the drive in diploids is from a previous study ${ }^{61}$.

To assess the reasons for the female fertility homing suppression drive's similar performance in haplodiploids and diploids despite differences in genetic load and rate of increase, we assessed panmictic population properties when the drive had reached $90 \%$ frequency with otherwise default parameters ${ }^{61}$ (see Table $\mathrm{S} 1$ and accompanying text). We found that the haplodiploid drive has a higher population of fertile females at $90 \%$ frequency compared to the diploid drive, thus reducing the chance that chasing can be initiated by stochastic escape of wild-type individuals from this region. This advantage can explain why the same drive in haplodiploids and diploids generally had similar performance, despite the greater power of the drive in diploids. 


\section{Discussion}

While modification type homing drives have previously been extensively analyzed in haplodiploid organisms ${ }^{51}$, this study investigates the details of how such species could be suppressed by a gene drive. Our results show that this is possible in haplodiploid organisms, and that a highly efficient drive can actually provide for very strong and successful suppression. This remains true even in more challenging environments involving continuous space where the "chasing" phenomenon can slow or prevent suppression.

In haplodiploid organisms, the number of options for suppression gene drives is greatly reduced compared to diploids. Previously developed sex-biasing strategies based on sex chromosomes such as X-shredders ${ }^{67}$ are certainly not possible, and even drives that don't rely on this will often lack the power needed to achieve high genetic loads. For example, homing drives targeting genes that are essential for both sexes ${ }^{68}$ or males would be eliminated quickly in males ${ }^{56,57}$, and even $\mathrm{TADE}^{69,70}$ drives would lack the power for suppression. Our modeling indicates that all of these drives, even in ideal form, would at best reach an intermediate allele frequency and be unable to impose a high genetic load on a population. Fortunately, the strategy of targeting a haplosufficient but essential female-specific gene with a homing drive was still able to achieve high genetic load (and though we modeled a female fertility gene, results would be broadly similar with a female viability gene). This type of gene drive has been well studied, with the latest drives even circumventing functional resistance alleles by careful target site selection ${ }^{8}$ or multiplexed gRNAs ${ }^{23}$. Both methods would likely also be needed to avoid functional resistance in large, natural haplodiploid population, but these methods should function with similar efficiency to their demonstrations in flies and mosquitoes, hence our decisions to exclude functional resistance alleles from this study. However, our study indicates that homing suppression drives in haplodiploids would need to keep somatic activity and embryo resistance allele formation even lower than in diploid drives to be effective. Though a rigorous requirement, there is still some flexibility in the specific values, and previously constructed drives have already achieved low enough somatic activity in flies ${ }^{23}$ and embryo resistance in mosquitoes ${ }^{8}$.

Surprisingly, we found that in continuous space models, female fertility homing suppression drives perform nearly as well in haplodiploid species as in diploids. Though more vulnerable to chasing over a fair amount of the parameter space we investigated, chases in most of this region were short, meaning that ultimate differences between the drives were minimal. Haplodiploid drives even had some minor advantages in some circumstances, such as reduced rates of drive loss and less loss of efficiency when the low-density growth rate was high. A previous study indicated that drives with less powerful mechanisms tended to be far more vulnerable to chasing ${ }^{61}$, but despite being unable to perform drive conversion in males to increase in frequency, this did not significantly apply to haplodiploid drives. This is perhaps because the drive can still diffuse quickly into wild-type populations but causes suppression more slowly than diploid drives, making it more difficult for pockets of wild-type individuals to stochastically avoid the drive in a chasing situation. 
Unlike TADE suppression drives, powerful homing drives are unconfined, even in haplodiploid organisms, which may be undesirable in some situations such as targeted suppression of invasive populations. Tethered drive systems could provide a solution to this ${ }^{71,72}$ and allow for confined drive in haplodiploids. Indeed, modeling shows that a TARE drive, which could be used to confine a homing drive that lacks Cas9, would perform well at an X-linked locus ${ }^{69}$, and it would have identical performance in haplodiploid species in other configurations ${ }^{70}$ as well.

X-linked homing drives in diploids would also have similar performance to drives in haplodiploids $^{51}$, and our modeling is thus equally applicable to both. Normally, we would expect autosomal diploid suppression drives to have equal or superior performance to X-linked drives. However, our modeling in continuous space indicates that there may be a narrow parameter space where an X-linked system would be preferred for avoiding long-term chasing outcomes. Of course, more realistic species-specific modeling and knowledge of model parameters would be necessary before development of an X-linked suppression drive could confidently be recommended over an autosomal version.

While development of gene drives in new species remains difficult, our results are nonetheless promising for future control of haplodiploid pests. Thus, studies developing CRISPR knock-in techniques in haplodiploid pest species, as well as characterization of germline promoter elements, female-specific target genes, and gRNA promoters can be considered high priority. As with diploids, ecological field investigations and more advanced modeling studies are also needed for accurate outcome predictions of gene drive deployment in specific species and regions of interest.

\section{Acknowledgements}

Thanks to Samuel E. Champer and Isabel K. Kim for assistance with SLiM programming. This study was supported by laboratory startup funds from Peking University and the SLS-Qidong Innovation Fund.

\section{References}

1. Godfray HCJ, North A, Burt A. How driving endonuclease genes can be used to combat pests and disease vectors. BMC Biol. 15, 81, 2017.

2. Hay BA, Oberhofer G, Guo M. Engineering the composition and fate of wild populations with gene drive. Annu Rev Entomol, 66, annurev-ento-020117-043154, 2021.

3. Bier E. Gene drives gaining speed. Nat Rev Genet, 1-18, 2021.

4. Burt A, Crisanti A. Gene drive: Evolved and synthetic. ACS Chem Biol, 13, 343-346, 2018.

5. Dhole S, Lloyd AL, Gould F. Gene drive dynamics in natural populations: The importance 
of density dependence, space, and sex. Annu Rev Ecol Evol Syst, 51, 505-531, 2020.

6. Champer J, Buchman A, Akbari OS. Cheating evolution: engineering gene drives to manipulate the fate of wild populations. Nat Rev Genet, 17, 146-159, 2016.

7. Rode NO, Estoup A, Bourguet D, Courtier-Orgogozo V, Débarre F. Population management using gene drive: molecular design, models of spread dynamics and assessment of ecological risks. Conserv. Genet. 20, 671-690, 2019.

8. Kyrou K, Hammond AM, Galizi R, Kranjc N, Burt A, Beaghton AK, Nolan T, Crisanti A. A CRISPR-Cas9 gene drive targeting doublesex causes complete population suppression in caged Anopheles gambiae mosquitoes. Nat Biotechnol, 2018.

9. Simoni A, Hammond AM, Beaghton AK, Galizi R, Taxiarchi C, Kyrou K, Meacci D, Gribble M, Morselli G, Burt A, Nolan T, Crisanti A. A male-biased sex-distorter gene drive for the human malaria vector Anopheles gambiae. Nat Biotechnol, 1-7, 2020.

10. Gantz VM, Jasinskiene N, Tatarenkova O, Fazekas A, Macias VM, Bier E, James AA. Highly efficient Cas9-mediated gene drive for population modification of the malaria vector mosquito Anopheles stephensi. Proc Natl Acad Sci U S A, 112, E6736-E6743, 2015.

11. Carballar-Lejarazú R, Ogaugwu C, Tushar T, Kelsey A, Pham TB, Murphy J, Schmidt H, Lee Y, Lanzaro GC, James AA. Next-generation gene drive for population modification of the malaria vector mosquito, Anopheles gambiae. Proc Natl Acad Sci U S A, 117, 22805-22814, 2020.

12. Adolfi A, Gantz VM, Jasinskiene N, Lee HF, Hwang K, Terradas G, Bulger EA, Ramaiah A, Bennett JB, Emerson JJ, Marshall JM, Bier E, James AA. Efficient population modification gene-drive rescue system in the malaria mosquito Anopheles stephensi. Nat Commun, 11, 1-13, 2020.

13. Teem JL, Alphey L, Descamps S, Edgington MP, Edwards O, Gemmell N, HarveySamuel T, Melnick RL, Oh KP, Piaggio AJ, Saah JR, Schill D, Thomas P, Smith T, Roberts A. Genetic biocontrol for invasive species. Front Bioeng Biotechnol, 8, 452, 2020.

14. Scott MJ, Gould F, Lorenzen M, Grubbs N, Edwards O, O’Brochta D. Agricultural production: assessment of the potential use of Cas9-mediated gene drive systems for agricultural pest control. J Responsible Innov, 5, S98-S120, 2018.

15. Shapiro RS, Chavez A, Porter CBM, Hamblin M, Kaas CS, DiCarlo JE, Zeng G, Xu X, Revtovich A V., Kirienko N V., Wang Y, Church GM, Collins JJ. A CRISPR-Cas9-based gene drive platform for genetic interaction analysis in Candida albicans. Nat Microbiol, 3, 73-82, 2018.

16. DiCarlo JE, Chavez A, Dietz SL, Esvelt KM, Church GM. Safeguarding CRISPR-Cas9 gene drives in yeast. Nat Biotechnol, 33, 1250-1255, 2015.

17. Roggenkamp E, Giersch RM, Schrock MN, Turnquist E, Halloran M, Finnigan GC. Tuning CRISPR-Cas9 gene drives in Saccharomyces cerevisiae. G3, 8, 999-1018, 2018.

18. Yan Y, Finnigan GC. Development of a multi-locus CRISPR gene drive system in budding yeast. Sci Rep, 8, 17277, 2018.

19. Champer J, Chung J, Lee YL, Liu C, Yang E, Wen Z, Clark AG, Messer PW. Molecular safeguarding of CRISPR gene drive experiments. Elife, 8, 2019.

20. Champer J, Wen Z, Luthra A, Reeves R, Chung J, Liu C, Lee YL, Liu J, Yang E, Messer PW, Clark AG. CRISPR gene drive efficiency and resistance rate is highly heritable with no common genetic loci of large effect. Genetics, 2019. 
21. Terradas G, Buchman AB, Bennett JB, Shriner I, Marshall JM, Akbari OS, Bier E. Inherently confinable split-drive systems in Drosophila. Nat Commun, 12, 1-12, 2021.

22. Kandul NP, Liu J, Bennett JB, Marshall JM, Akbari OS. A confinable home-and-rescue gene drive for population modification. Elife, 10, 2021.

23. Yang E, Metzloff M, Langmüller AM, Clark AG, Messer PW, Champer J. A homing suppression gene drive with multiplexed gRNAs maintains high drive conversion efficiency and avoids functional resistance alleles. bioRxiv, 2021.05.27.446071, 2021.

24. Reeves RG, Bryk J, Altrock PM, Denton JA, Reed FA. First steps towards underdominant genetic transformation of insect populations. PLoS One, 9, e97557, 2014.

25. Oberhofer G, Ivy T, Hay BA. Cleave and Rescue, a novel selfish genetic element and general strategy for gene drive. Proc Natl Acad Sci, 201816928, 2019.

26. Champer J, Lee E, Yang E, Liu C, Clark AG, Messer PW. A toxin-antidote CRISPR gene drive system for regional population modification. Nat Commun, 11, 1082, 2020.

27. Oberhofer G, Ivy T, Hay BA. Gene drive and resilience through renewal with next generation Cleave and Rescue selfish genetic elements. Proc Natl Acad Sci U S A, 117, 9013-9021, 2020.

28. Maselko M, Feltman N, Upadhyay A, Hayward A, Das S, Myslicki N, Peterson AJ, O’Connor MB, Smanski MJ. Engineering multiple species-like genetic incompatibilities in insects. Nat Commun, 11, 1-7, 2020.

29. Champer SE, Oh SY, Liu C, Wen Z, Clark AG, Messer PW, Champer J. Computational and experimental performance of CRISPR homing gene drive strategies with multiplexed gRNAs. Sci Adv, 6, eaaz0525, 2020.

30. Champer J, Liu J, Oh SY, Reeves R, Luthra A, Oakes N, Clark AG, Messer PW. Reducing resistance allele formation in CRISPR gene drive. Proc Natl Acad Sci, 115, 5522-5527, 2018.

31. KaramiNejadRanjbar M, Eckermann KN, Ahmed HMM, Sánchez C. HM, Dippel S, Marshall JM, Wimmer EA. Consequences of resistance evolution in a Cas9-based sexconversion suppression gene drive for insect pest management. Proc Natl Acad Sci, $201713825,2018$.

32. Champer J, Reeves R, Oh SY, Liu C, Liu J, Clark AG, Messer PW. Novel CRISPR/Cas9 gene drive constructs reveal insights into mechanisms of resistance allele formation and drive efficiency in genetically diverse populations. PLoS Genet, 13, e1006796, 2017.

33. Xu X-RS, Bulger EA, Gantz VM, Klanseck C, Heimler SR, Auradkar A, Bennett JB, Miller LA, Leahy S, Juste SS, Buchman A, Akbari OS, Marshall JM, Bier E. Active genetic neutralizing elements for halting or deleting gene drives. Mol Cell, 2020.

34. López Del Amo V, Bishop AL, Sánchez C HM, Bennett JB, Feng X, Marshall JM, Bier E, Gantz VM. A transcomplementing gene drive provides a flexible platform for laboratory investigation and potential field deployment. Nat Commun, 11, 1-12, 2020.

35. Champer J, Yang E, Lee E, Liu J, Clark AG, Messer PW. A CRISPR homing gene drive targeting a haplolethal gene removes resistance alleles and successfully spreads through a cage population. Proc Natl Acad Sci, 117, 24377-24383, 2020.

36. Oberhofer G, Ivy T, Hay BA. Behavior of homing endonuclease gene drives targeting genes required for viability or female fertility with multiplexed guide RNAs. Proc Natl Acad Sci, 115, E9343-E9352, 2018.

37. Grunwald HA, Gantz VM, Poplawski G, Xu X-RS, Bier E, Cooper KL. Super-Mendelian inheritance mediated by CRISPR-Cas9 in the female mouse germline. Nature, 566, 105- 
$109,2019$.

38. Hammond AM, Kyrou K, Bruttini M, North A, Galizi R, Karlsson X, Kranjc N, Carpi FM, D'Aurizio R, Crisanti A, Nolan T. The creation and selection of mutations resistant to a gene drive over multiple generations in the malaria mosquito. PLOS Genet, 13, e1007039, 2017.

39. Pham TB, Phong CH, Bennett JB, Hwang K, Jasinskiene N, Parker K, Stillinger D, Marshall JM, Carballar-Lejarazú R, James AA. Experimental population modification of the malaria vector mosquito, Anopheles stephensi. PLOS Genet, 15, e1008440, 2019.

40. Unckless RL, Clark AG, Messer PW. Evolution of resistance against CRISPR/Cas9 gene drive. Genetics, 205, 827-841, 2017.

41. Willis K, Burt A. Double drives and private alleles for localised population genetic control. PLOS Genet, 17, e1009333, 2021.

42. Gomulkiewicz R, Thies ML, Bull JJ. Evading resistance to gene drives. Genetics, 217 , 2021.

43. Noble C, Olejarz J, Esvelt K, Church G, Nowak M. Evolutionary dynamics of CRISPR gene drives. Sci Adv, 3, e1601964, 2017.

44. Edgington MP, Harvey-Samuel T, Alphey L. Population-level multiplexing, a promising strategy to manage the evolution of resistance against gene drives targeting a neutral locus. Evol Appl, eva.12945, 2020.

45. Rode NO, Courtier-Orgogozo V, Débarre F. Can a population targeted by a CRISPRbased homing gene drive Be rescued? G3 Genes|Genomes|Genetics, 10, 3403-3415, 2020.

46. Beaghton AK, Hammond A, Nolan T, Crisanti A, Burt A. Gene drive for population genetic control: non-functional resistance and parental effects. Proceedings Biol Sci, 286, 20191586, 2019.

47. Lambert B, North A, Burt A, Godfray HCJ. The use of driving endonuclease genes to suppress mosquito vectors of malaria in temporally variable environments. Malar J, 17, 154, 2018.

48. Prowse TAA, Cassey P, Ross J V, Pfitzner C, Wittmann TA, Thomas P. Dodging silver bullets: good CRISPR gene-drive design is critical for eradicating exotic vertebrates. Proceedings Biol Sci, 284, 20170799, 2017.

49. Sánchez C. HM, Wu SL, Bennett JB, Marshall JM. MGD <scp>riv</scp> E: A modular simulation framework for the spread of gene drives through spatially explicit mosquito populations. Methods Ecol Evol, 2041-210X.13318, 2019.

50. Wu SL, Bennett JB, Sánchez C. HM, Dolgert AJ, León TM, Marshall JM. MGDrivE 2: A simulation framework for gene drive systems incorporating seasonality and epidemiological dynamics. PLOS Comput Biol, 17, e1009030, 2021.

51. Li J, Aidlin Harari O, Doss A, Walling LL, Atkinson PW, Morin S, Tabashnik BE. Can CRISPR gene drive work in pest and beneficial haplodiploid species? Evol Appl, eva.13032, 2020.

52. Vinson SB. Impact of the invasion of the imported fire ant. Insect Sci, 20, 439-455, 2013.

53. Wang L, Lu Y. Red Imported Fire Ant <Emphasis Type="Italic" $>$ Solenopsis invicta</Emphasis> Buren. Biol Invasions Its Manag China, 11, 299-315, 2017.

54. Lee CM, Lee D-S, Kwon T-S, Athar M, Park Y-S. Predicting the Global Distribution of Solenopsis geminata (Hymenoptera: Formicidae) under Climate Change Using the MaxEnt Model. Insects, 12, 229, 2021. 
55. Chiu Y-K, Hsu J-C, Chang T, Huang Y-C, Wang J. Mutagenesis mediated by CRISPR/Cas9 in the red imported fire ant, Solenopsis invicta. Insectes Soc, 67, 317-326, 2020.

56. Faber NR, Meiborg AB, McFarlane GR, Gorjanc G, Harpur BA. A gene drive does not spread easily in populations of the honey bee parasite Varroa destructor. bioRxiv, 2021.04.30.442149, 2021.

57. Lester PJ, Bulgarella M, Baty JW, Dearden PK, Guhlin J, Kean JM. The potential for a CRISPR gene drive to eradicate or suppress globally invasive social wasps. Sci Rep, 10, 1-13, 2020.

58. Bull JJ, Remien CH, Krone SM. Gene-drive-mediated extinction is thwarted by population structure and evolution of sib mating. Evol Med public Heal, 2019, 66-81, 2019.

59. North AR, Burt A, Godfray HCJ. Modelling the potential of genetic control of malaria mosquitoes at national scale. BMC Biol, 17, 26, 2019.

60. North AR, Burt A, Godfray HCJ. Modelling the suppression of a malaria vector using a CRISPR-Cas9 gene drive to reduce female fertility. BMC Biol, 18, 98, 2020.

61. Champer J, Kim IK, Champer SE, Clark AG, Messer PW. Suppression gene drive in continuous space can result in unstable persistence of both drive and wild-type alleles. Mol Ecol, 30, 1086-1101, 2021.

62. Faber NR, McFarlane GR, Gaynor RC, Pocrnic I, Whitelaw CBA, Gorjanc G. Novel combination of CRISPR-based gene drives eliminates resistance and localises spread. Sci Rep, 11, 3719, 2021.

63. Haller BC, Messer PW. SLiM 3: Forward genetic simulations beyond the Wright-Fisher model. Mol Biol Evol, 36, 632-637, 2019.

64. Hammond A, Karlsson X, Morianou I, Kyrou K, Beaghton A, Gribble M, Kranjc N, Galizi R, Burt A, Crisanti A, Nolan T. Regulating the expression of gene drives is key to increasing their invasive potential and the mitigation of resistance. PLOS Genet, 17, e1009321, 2021.

65. Hammond A, Galizi R, Kyrou K, Simoni A, Siniscalchi C, Katsanos D, Gribble M, Baker D, Marois E, Russell S, Burt A, Windbichler N, Crisanti A, Nolan T. A CRISPR-Cas9 gene drive system targeting female reproduction in the malaria mosquito vector Anopheles gambiae. Nat Biotechnol, 34, 78-83, 2015.

66. Eckhoff PA, Wenger EA, Godfray HC, Burt A. Impact of mosquito gene drive on malaria elimination in a computational model with explicit spatial and temporal dynamics. Proc Natl Acad Sci U S A, 114, E255-e264, 2017.

67. Galizi R, Doyle LA, Menichelli M, Bernardini F, Deredec A, Burt A, Stoddard BL, Windbichler N, Crisanti A. A synthetic sex ratio distortion system for the control of the human malaria mosquito. Nat Commun, 5, 3977, 2014.

68. Burt A. Site-specific selfish genes as tools for the control and genetic engineering of natural populations. Proc Biol Sci, 270, 921-928, 2003.

69. Champer J, Kim IK, Champer SE, Clark AG, Messer PW. Performance analysis of novel toxin-antidote CRISPR gene drive systems. BMC Biol, 18, 27, 2020.

70. Champer J, Champer SE, Kim IK, Clark AG, Messer PW. Design and analysis of CRISPR-based underdominance toxin-antidote gene drives. Evol Appl, eva.13180, 2020.

71. Dhole S, Lloyd AL, Gould F. Tethered homing gene drives: A new design for spatially restricted population replacement and suppression. Evol Appl, eva.12827, 2019. 
bioRxiv preprint doi: https://doi.org/10.1101/2021.10.12.464047; this version posted October 13,2021 . The copyright holder for this preprint (which was not certified by peer review) is the author/funder, who has granted bioRxiv a license to display the preprint in perpetuity. It is made available under aCC-BY-NC 4.0 International license.

72. Metzloff M, Yang E, Dhole S, Clark AG, Messer PW, Champer J. Experimental demonstration of tethered gene drive systems for confined population modification or suppression. bioRxiv, 2021.05.29.446308, 2021. 


\section{Supplemental Information}

\section{Supplemental Results}
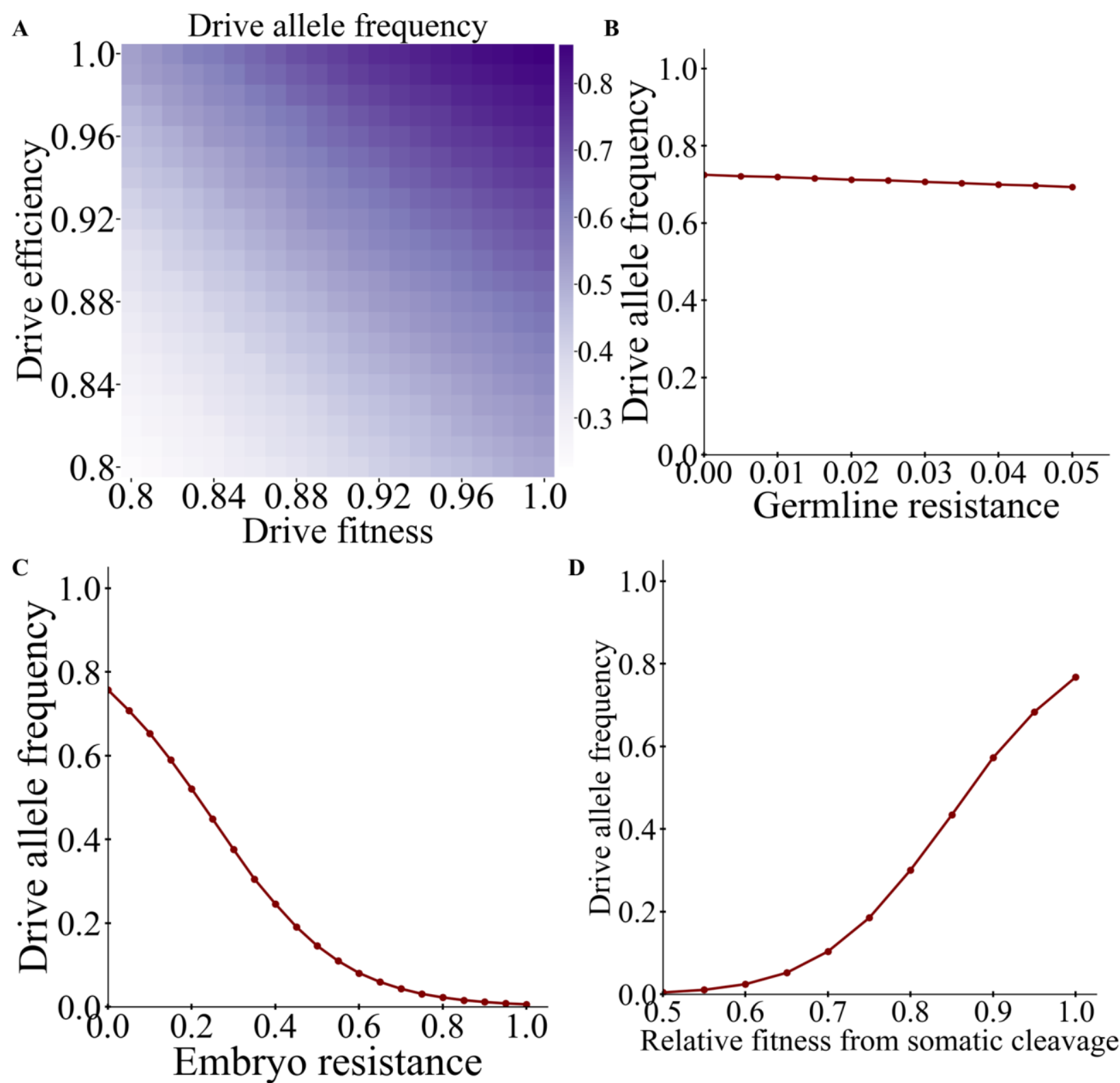

Figure S1 Effects of performance characteristics on haplodiploid drive frequency at generation 16. Drive heterozygotes (and drive males for the haplodiploid drive) with default parameters were released at $1 \%$ frequency into a panmictic population of wild-type individuals. The drive allele frequency is displayed 16 generation later for varying (A) efficiency and homozygote fitness, $(\mathbf{B})$ germline resistance allele formation rate, $(\mathbf{C})$ embryo resistance allele formation rate, or (D) fitness cost in female heterozygotes due to somatic CRISPR cleavage activity. Displayed data is the average from 20 (A) or 200 (B-C) simulations. 
A

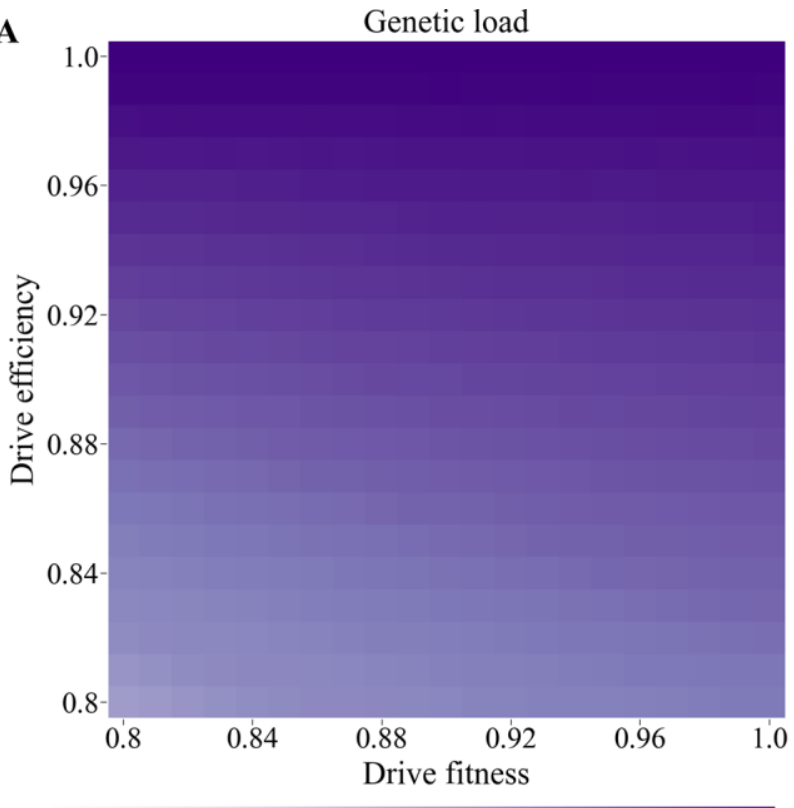

B

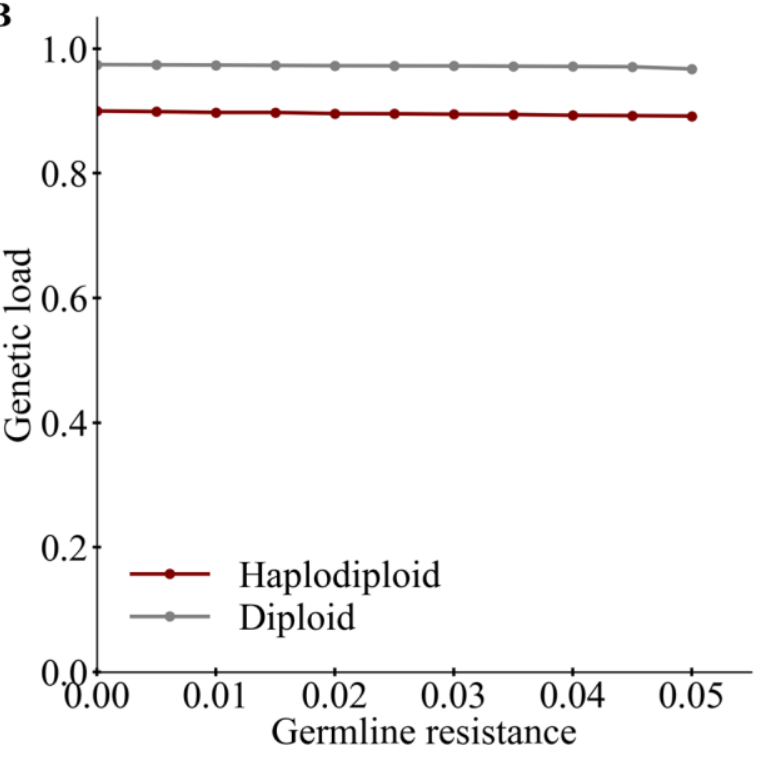

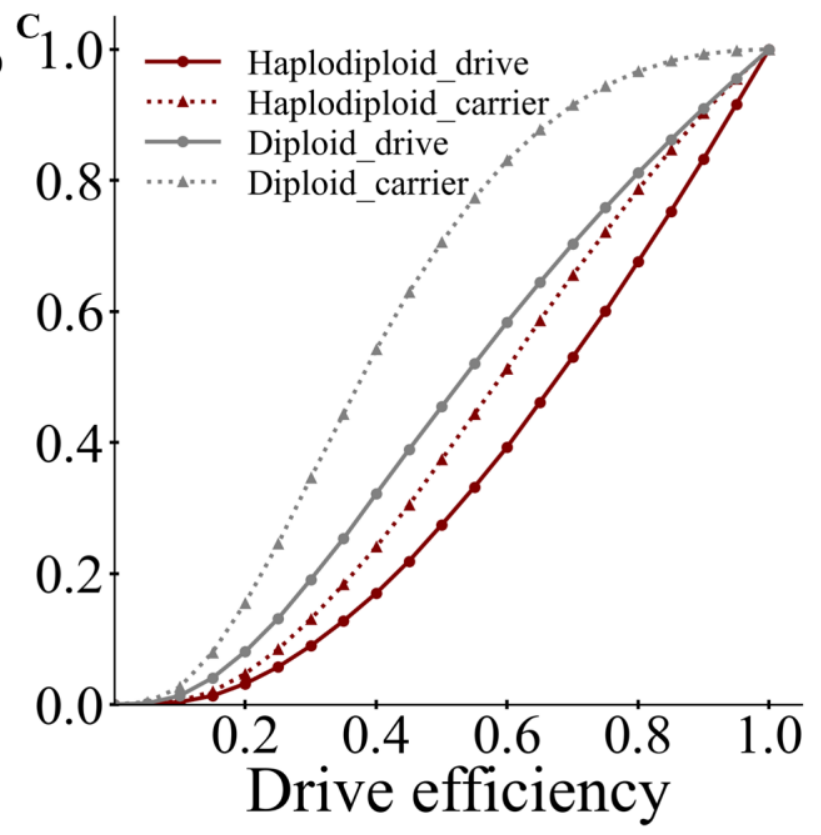

Figure S2 Effects of additional performance characteristics on the genetic load and drive equilibrium frequencies. The genetic load for female fertility homing suppression drives at equilibrium is displayed for drives with default performance characteristics except for (A) varying drive efficiency and homozygote fitness or (B) varying germline resistance allele formation rate. (C) Also displayed is the drive allele and carrier frequencies for both haplodiploid and diploid drives at equilibrium when maximum genetic load has been reached. Displayed data is the average from (A,C) 20 or (B) 200 simulations per data point. 
A

1.0

Drive lost without chasing

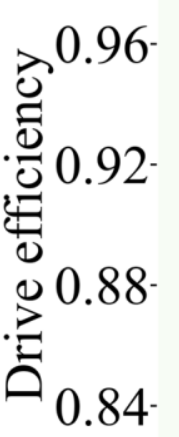

0.8

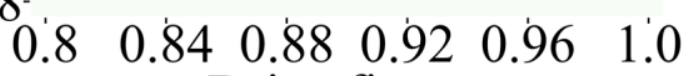

Drive fitness

C 0.06

Drive lost without chasing

$\sum_{0.02}^{0.05}$

D

0.06

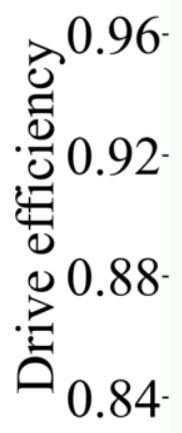

Drive lost after chasing

0.8

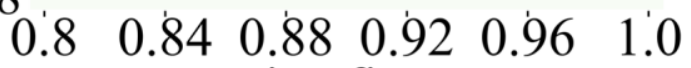
Drive fitness Drive lost after chasing

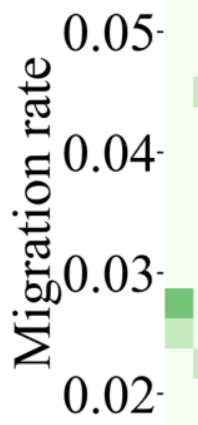

0.01

0.01 $\begin{array}{llllll}2.0 & 4.0 & 6.0 & 8.0 & 10.0 & 12.0\end{array}$ Low density growth rate Low density growth rate

0.00

0.05

0.10

0.15

0.20

Figure S3 Drive loss outcomes in continuous space. Female drive heterozygotes and drive males were released in a 0.01 radius circle into the middle of a spatial population of 50,000 individuals and were tracked for 1,000 generations. Default parameters were used with $(\mathbf{A}, \mathbf{B})$ varying drive efficiency and fitness of $(\mathbf{C}, \mathbf{D})$ varying migration rate and low-density growth rate. Rates of drive loss $(\mathbf{A}, \mathbf{C})$ without chasing or $(\mathbf{B , D})$ after chasing are displayed. 20 simulations were assessed for each point in the parameter space. 

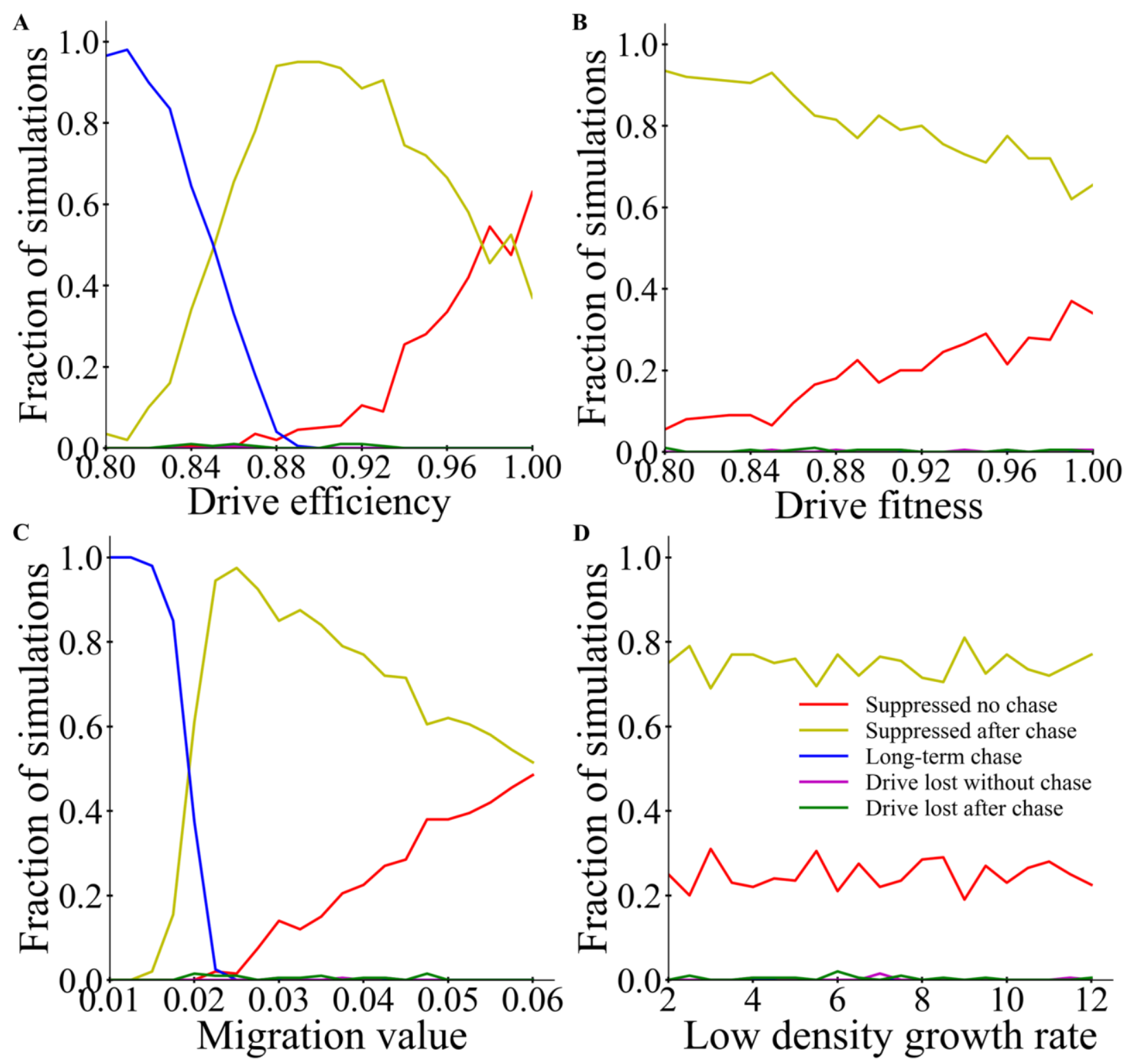

Figure S4 Outcomes of a drive release in continuous space. Female drive heterozygotes and drive males with default parameters and varying $(\mathbf{A})$ efficiency, $(\mathbf{B})$ fitness, $(\mathbf{C})$ migration rate, and $(\mathbf{D})$ low-density growth rate were released in a 0.01 radius circle into the middle of a spatial population of 50,000 individuals and were tracked for 1,000 generations. The rate of each outcome type is displayed. 200 simulations were assessed for each point in the parameter space. 


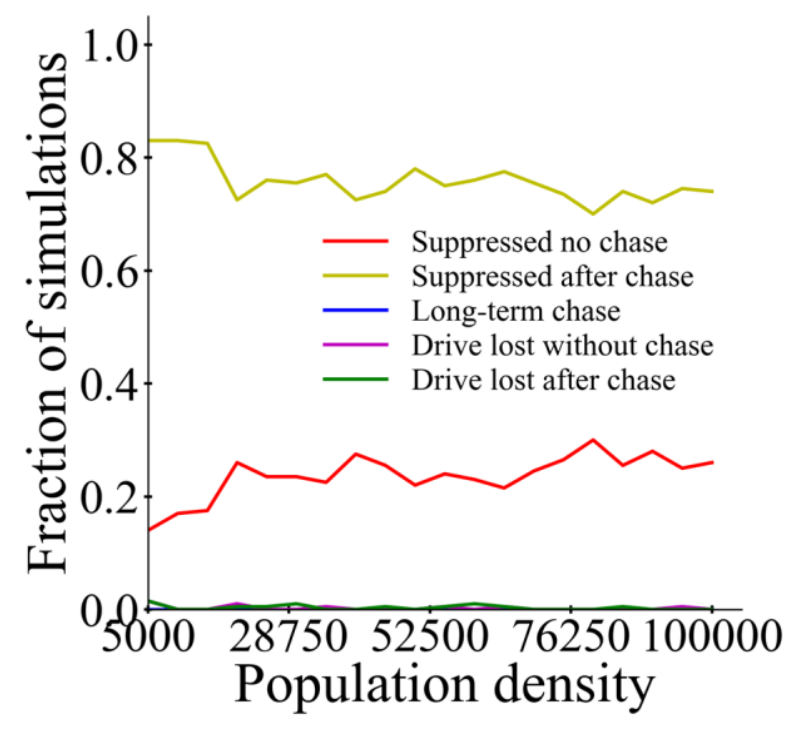

Figure S5 Effect of population density on drive outcomes in continuous space. Female drive heterozygotes and drive males with default parameters were released in a 0.01 radius circle into the middle of a spatial population of and were tracked for 1,000 generations. The rate of each outcome type is displayed for varying population density. 200 simulations were assessed for each point in the parameter space. 

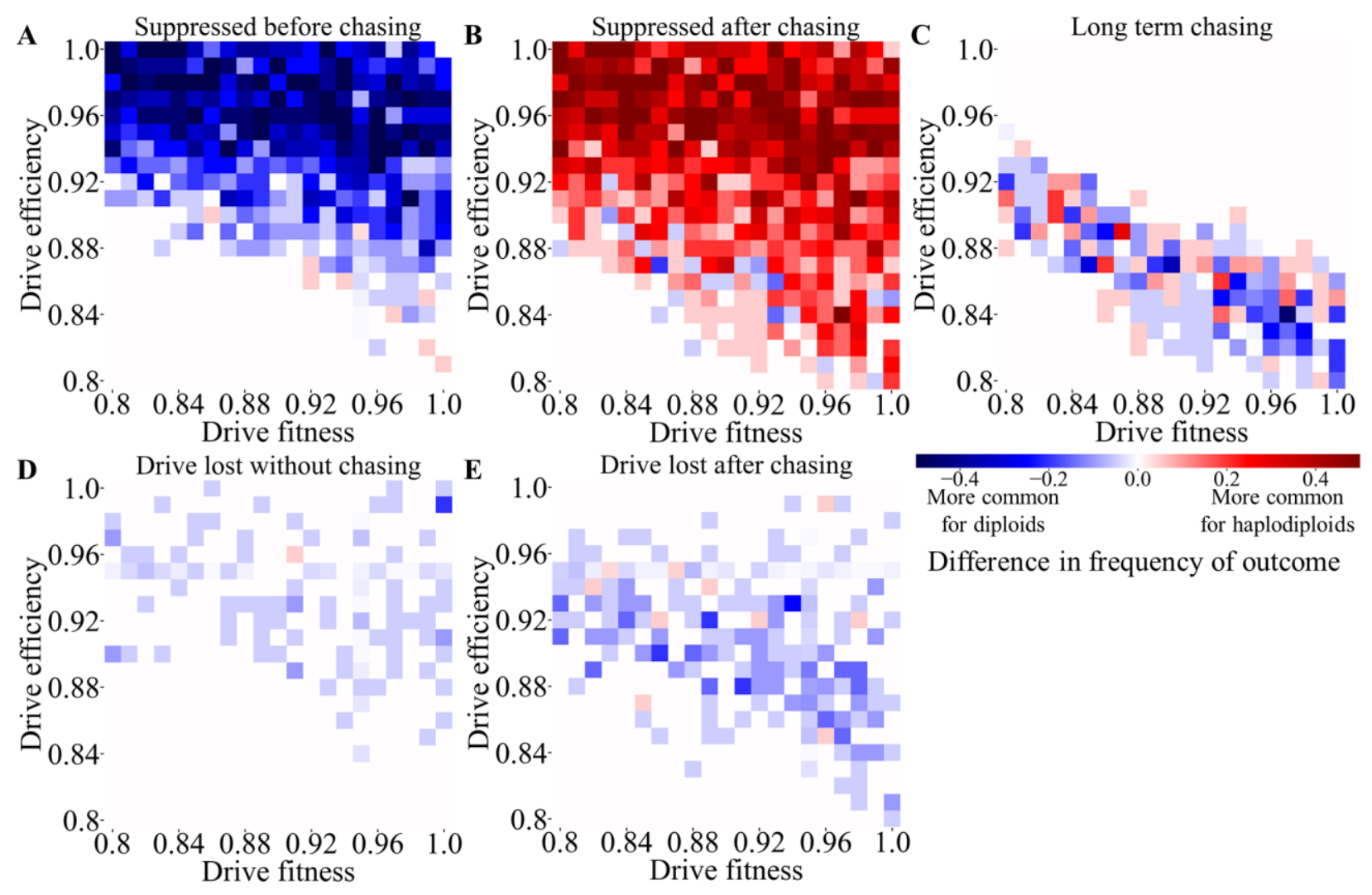

Figure S6 Comparison of continuous space outcomes between haplodiploids and diploids with varying drive efficiency and fitness. Drive heterozygotes (and drive males for the haplodiploid drive) with default parameters and varying drive efficiency and fitness were released in a 0.01 radius circle into the middle of a spatial population of 50,000 individuals and were tracked for 1,000 generations. Differences in outcome rates between haplodiploids and diploids (red is more common in haplodiploids, and blue is more common in diploids) are displayed for (A) rapid suppression without chasing, (B) suppression after a period of chasing, (C) simulations in which chasing was still occurring at the end of the simulation, (D) drive loss without chasing, and (E) drive loss after a period of chasing. 20 simulations were assessed for each point in the parameter space. Data for diploid individuals is from a previous study ${ }^{61}$. 

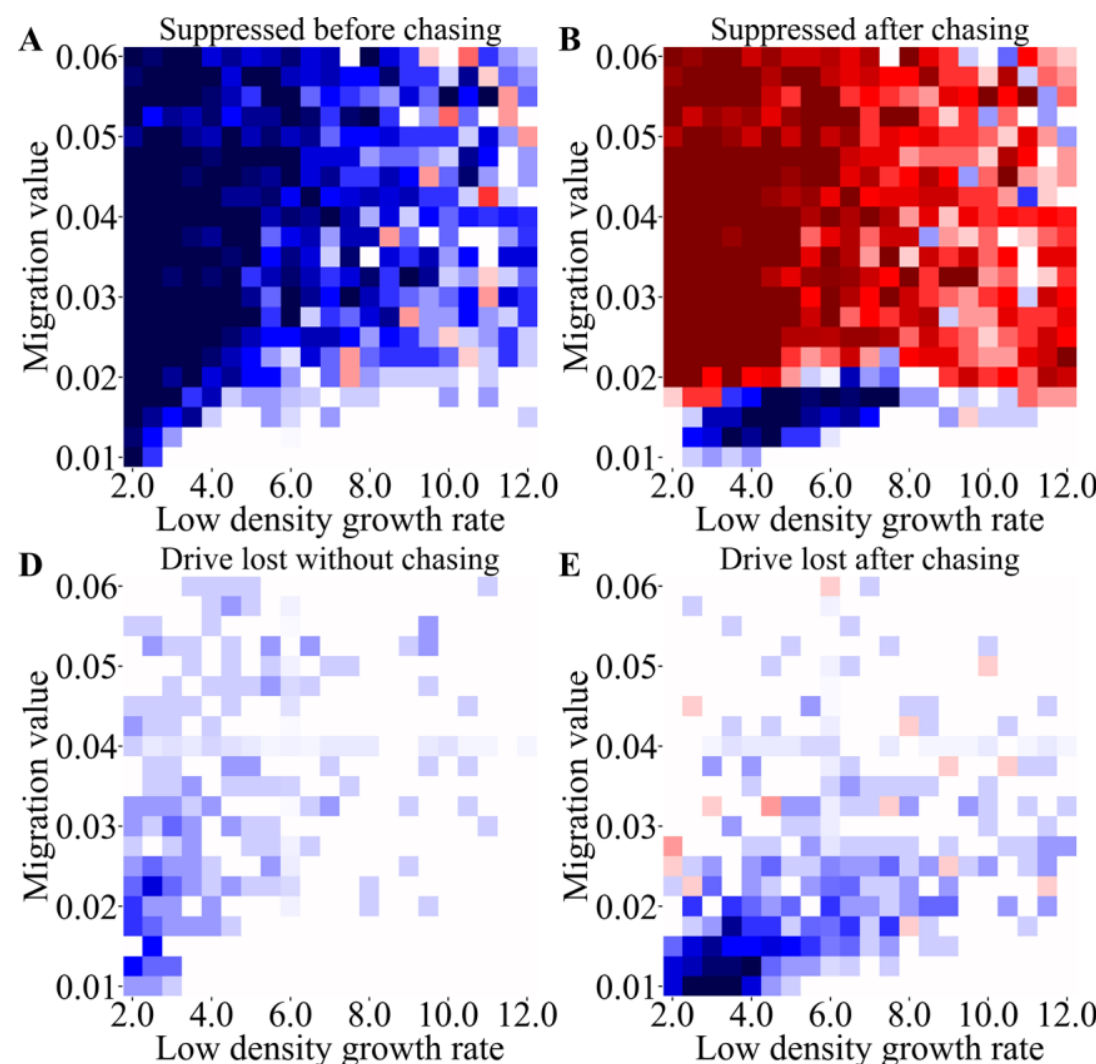

C 0.06 Long term chasing
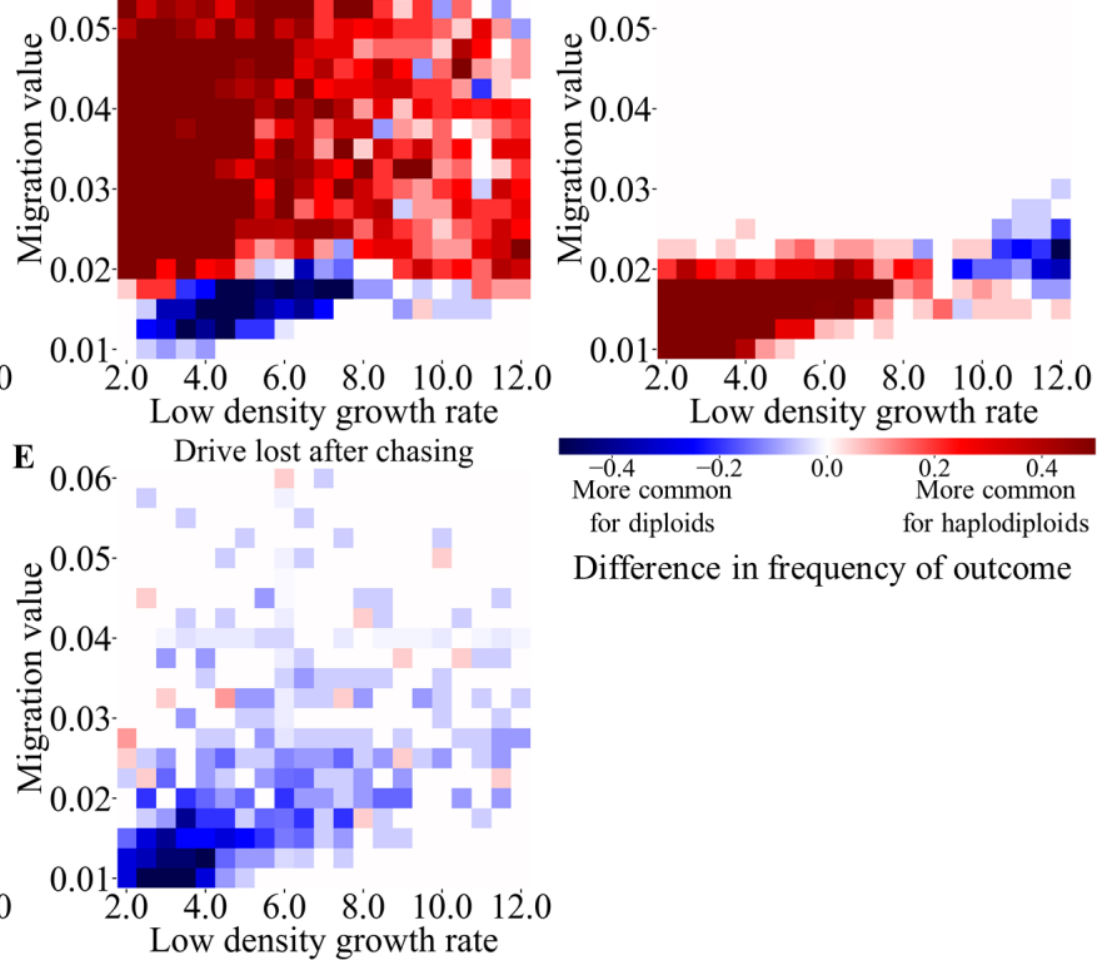

Difference in frequency of outcome

Figure S7 Comparison of continuous space outcomes between haplodiploids and diploids with varying migration and low-density growth rate. Drive heterozygotes (and drive males for the haplodiploid drive) with default drive performance parameters and varying migration and low-density growth rate were released in a 0.01 radius circle into the middle of a spatial population of 50,000 individuals and were tracked for 1,000 generations. Differences in outcome rates between haplodiploids and diploids (red is more common in haplodiploids, and blue is more common in diploids) are displayed for (A) rapid suppression without chasing, (B) suppression after a period of chasing, (C) simulations in which chasing was still occurring at the end of the simulation, (D) drive loss without chasing, and (E) drive loss after a period of chasing. 20 simulations were assessed for each point in the parameter space. Data for diploid individuals is from a previous study ${ }^{61}$. 
Table S1 Population characteristics at $90 \%$ drive frequency correlate with chasing

\begin{tabular}{|l|c|c|c|c|c|}
\hline & $\begin{array}{c}\text { Haplodiploid } \\
\text { female fertility } \\
\text { homing }\end{array}$ & $\begin{array}{c}\text { Female } \\
\text { fertility } \\
\text { homing }\end{array}$ & $\begin{array}{c}\text { Both-sex } \\
\text { fertility } \\
\text { homing }\end{array}$ & $\begin{array}{c}\text { Driving Y } \\
\text { (X-shredder) }\end{array}$ & $\begin{array}{c}\text { TADS } \\
\text { Suppression }\end{array}$ \\
\hline Population size & 3404 & 1363 & 865 & 10969 & 10999 \\
\hline Fertile females & $10 \%$ & $7.9 \%$ & $8.1 \%$ & $9.1 \%$ & $50 \%$ \\
\hline Sterile females & $40 \%$ & $43 \%$ & $41 \%$ & N/A & N/A \\
\hline Fertile males & $50 \%$ & $49 \%$ & $8.8 \%$ & $8.2 \%$ & $8.3 \%$ \\
\hline Sterile males & N/A & N/A & $42 \%$ & $83 \% *^{*}$ & $42 \%$ \\
\hline $\begin{array}{l}\text { Expected } \\
\text { reproducing } \\
\text { females }\end{array}$ & $\mathbf{3 4 5}$ & $\mathbf{1 1 4}$ & $\mathbf{1 2}$ & $\mathbf{1 0 3}^{* *}$ & $\mathbf{9 3 4}$ \\
\hline Chasing rate & medium & medium & very high & high & low \\
\hline
\end{tabular}

Values are the average over 20 simulations. Except for the haplodiploid drive, data is from a previous study ${ }^{61}$. *Drive-carrying males. **Includes only females expected to mate with wildtype males.

The table shows panmictic population characteristics when the drive reaches $90 \%$ allele frequency in the population (using default parameters for each drive). In a spatial model, this corresponds to inner edge of the drive wave of advance where population suppression is imminent. If wild-type individuals can generate offspring around here while avoiding the drive, a stochastic process, they can move into adjacent empty areas to begin a "chase". Higher population sizes tend to reduce the frequency of this process. Thus, the both-sex fertility homing drive with only 12 reproducing females is highly prone to chasing compared to the female fertility homing drive, while the TADS suppression drive with 934 is substantially less prone to chasing. The Driving Y has a similar number of reproducing females that would be expected to generate daughters, but its total population size is overall higher, so these individuals would have fewer offspring and thus promote chasing. The haplodiploid female fertility drive, according to this analysis, would thus be somewhat better than the diploid drive at avoiding chasing, but other factors that likely affect chasing as well (particularly overall drive power/genetic load and rate of increase) result in broadly similar performance between the two drives. 